The Astrophysical Journal, 651:994-1004, 2006 November 10

(C) 2006. The American Astronomical Society. All rights reserved. Printed in U.S.A.

\title{
EARLY TIME CHROMATIC VARIATIONS IN THE WIND-SWEPT MEDIUM OF GRB 021211 AND THE FAINTNESS OF ITS AFTERGLOW
}

\author{
M. C. Nysewander, ${ }^{1}$ D. E. Reichart, ${ }^{1}$ H.-S. Park, ${ }^{2}$ G. G. Williams, ${ }^{3}$ K. Kinugasa, ${ }^{4}$ D. Q. Lamb, ${ }^{5}$ A. A. Henden, ${ }^{6}$ \\ S. Klose ${ }^{7}$ T. Kato, ${ }^{8}$ A. Harper,${ }^{5}$ H. Yamaoka, ${ }^{9}$ C. Laws, ${ }^{10}$ K. ToriI, ${ }^{11}$ D. G. York, ${ }^{5}$ J. C. Barentine, ${ }^{12}$ \\ J. Dembicky, ${ }^{12}$ R. J. McMillan, ${ }^{12}$ J. A. Moran, ${ }^{1}$ D. H. Hartmann, ${ }^{13}$ B. Ketzeback, ${ }^{12}$ M. B. Bayliss, ${ }^{1}$ \\ J. W. Bartelme, ${ }^{1}$ J. A. Crain, ${ }^{1}$ A. C. Foster, ${ }^{1}$ M. Schwartz ${ }^{14}$ P. Holvorcem, ${ }^{14}$ P. A. Price, ${ }^{15}$ \\ R. Canterna, ${ }^{16}$ G. B. Crew, ${ }^{17}$ G. R. Ricker, ${ }^{17}$ and S. D. Barthelmy ${ }^{18}$ \\ Received 2005 May 23; accepted 2006 June 15
}

\begin{abstract}
We present Follow-Up Network for Gamma-Ray Bursts (FUN GRB) Collaboration observations of the optical afterglow of GRB 021211 made between $143 \mathrm{~s}$ and 102 days after the burst. Our unique data set includes the earliest filtered detections and color information for an afterglow in the pre-Swift era. We find that the afterglow is best described by (1) a propagation through a wind-swept medium, (2) a cooling break that is blueward of the observed optical frequencies, and (3) a hard electron energy distribution. However, superimposed on this "standard model" behavior we find, one and possibly two, significant chromatic variations during the first few hours after the burst. We consider possible reasons for these variations, including the possibility that they are due to a dust echo. Finally, we constrain physical parameters that describe the afterglow and the surrounding medium for a variety of scenarios and find that GRB 021211 's afterglow is faint for a combination of reasons: (1) a low fraction of energy in relativistic electrons, (2) a low density for the wind-swept medium, implying either a low mass-loss rate and/or a high wind velocity for the progenitor, (3) a wide opening/viewing angle for the jet, and possibly (4) moderate source-frame extinction. The jet appears to be significantly far from equipartition and magnetically dominated. More extreme versions of this might explain the darkness of many afterglows in the Swift era.
\end{abstract}

Subject headings: dust, extinction — gamma rays: bursts — magnetic fields — scattering — stars: winds, outflows — stars: Wolf-Rayet

\section{INTRODUCTION}

Discovery of gamma-ray burst (GRB) afterglows has become almost commonplace. However, we are still in a regime where nearly every well-sampled afterglow contributes to our understanding of the phenomenon in new and meaningful ways. Ob-

\footnotetext{
${ }^{1}$ Department of Physics and Astronomy, University of North Carolina at Chapel Hill, Campus Box 3255, Chapel Hill, NC 27599; mnysewan@physics .unc.edu, reichart@physics.unc.edu.

${ }^{2}$ Lawerence Livermore National Laboratory, 7000 East Avenue, Livermore, CA 94550 .

3 MMT Observatory, University of Arizona, Tucson, AZ 85721.

${ }^{4}$ Gunma Astronomical Observatory, 6860-86 Nakayama Takayama, Agatsuma, Gunma 377-0702, Japan.

5 Department of Astronomy and Astrophysics, University of Chicago, 5640 South Ellis Avenue, Chicago, IL 60615.

${ }^{6}$ American Association of Variable Star Observers, Clinton B. Ford Astronomical Data and Research Center, 25 Birch Street, Cambridge, MA 02138.

7 Thüringer Landessternwarte, 07778 Tautenburg, Germany.

8 Department of Astronomy, Faculty of Science, Kyoto University, Sakyo-ku, Kyoto 606-8502, Japan.

9 Department of Physics, Kyushu University, Fukuoka 810-8560, Japan.

10 Department of Astronomy, Box 351580, University of Washington, Seattle, WA 98195.

${ }_{11}$ Department of Earth and Space Science, Graduate School of Science, 1-1 Machikaneyama-cho, Toyonaka, Osaka 560-0043, Japan.

${ }_{12}$ Apache Point Observatory, P.O. Box 59, Sunspot, NM 88349

13 Department of Physics and Astronomy, Clemson University, Clemson, SC 29634.

${ }_{14}$ Tenagra Observatory, HC2 Box 292, Nogales, AZ 85621.

15 Institute of Astronomy, University of Hawaii, 2680 Woodlawn Drive, Honolulu, HI 96822-1897.

16 Department of Physics and Astronomy, University of Wyoming, P.O. Box 3905, Laramie, WY 82072.

${ }_{17}$ Center for Space Research, Massachusetts Institute of Technology, 70 Vassar Street, Cambridge, MA, 02139.

${ }_{18}$ NASA Goddard Space Flight Center, Code 661, Greenbelt, MD 20771.
}

servationally, GRB 021211 distinguishes itself in two ways: (1) it is the second GRB for which an optical afterglow was observed within minutes of the burst, thanks to rapid responses by the High Energy Transient Explorer-2 (HETE-2) satellite (Crew et al. 2002, 2003) and three robotic telescopes - the Rapid Telescope for Optical Response (RAPTOR; Wozniak et al. 2002); the Katzman Automatic Imaging Telescope (KAIT; Li et al. 2002, 2003); and the Super-Livermore Optical Transient Imaging System (SuperLOTIS; Park et al. 2002; this paper); and (2) it is the first GRB for which filtered detections (beginning $143 \mathrm{~s}$ after the burst) and color information (beginning 38 minutes after the burst) were obtained at early times.

In addition to observations presented in GRB Coordinates Network (GCN) Circulars, many groups have presented their observations in peer-reviewed journals: Li et al. (2003) present an unfiltered light curve beginning $105 \mathrm{~s}$ after the burst; Fox et al. (2003) present an unfiltered light curve beginning 21 minutes after the burst and filtered optical, NIR, and radio observations beginning $2.0 \mathrm{hr}$ after the burst; Pandey et al. (2003) present filtered optical observations beginning $6.8 \mathrm{hr}$ after the burst; Holland et al. (2004) present filtered optical and NIR observations of both the afterglow and host galaxy beginning $17 \mathrm{hr}$ after the burst and measure the spectral flux distribution of the afterglow around $21 \mathrm{hr}$ after the burst; and Smith et al. (2005) present submillimeter observations around $25 \mathrm{hr}$ and 10 days after the burst. Finally, Della Valle et al. (2003) present photometric and spectral evidence for an associated supernova at late times.

As in the case of GRB 990123 (Akerlof et al. 1999), the optical afterglow faded more rapidly at first, presumably due to a reverse shock (Wei 2003; Fox et al. 2003; Li et al. 2003; Holland et al. 2004). However, these afterglows differ in that GRB 021211 was $\approx 3-4$ mag fainter, despite a lower redshift 
TABLE 1

FUN GRB Collaboration Observations of the Afterglow of GRB 021211

\begin{tabular}{|c|c|c|c|c|}
\hline $\begin{array}{c}\text { Date } \\
\text { (UTC) }\end{array}$ & Mean $\Delta t$ & Filter & Magnitude $^{\mathrm{a}}$ & Telescope \\
\hline Dec $11.4732^{b}$ & 2.84 minutes & $R$ & $15.24 \pm 0.07$ & $0.60 \mathrm{~m}$ Super-LOTIS \\
\hline Dec $11.4751^{\mathrm{b}}$. & 5.63 minutes & $R$ & $16.26 \pm 0.12$ & $0.60 \mathrm{~m}$ Super-LOTIS \\
\hline Dec $11.4986 .$. & 39.4 minutes & $I_{\mathrm{C}}$ & $18.60 \pm 0.11$ & $0.81 \mathrm{~m}$ Tenagra II \\
\hline Dec $11.5114 \ldots \ldots \ldots \ldots \ldots \ldots$ & 57.9 minutes & $R_{\mathrm{C}}$ & $19.52 \pm 0.13$ & $0.81 \mathrm{~m}$ Tenagra II \\
\hline Dec $11.5239 \ldots \ldots \ldots \ldots \ldots \ldots$ & 75.9 minutes & $V$ & $20.06 \pm 0.41$ & $0.81 \mathrm{~m}$ Tenagra II \\
\hline Dec $11.5366 \ldots \ldots$ & 94.1 minutes & $B$ & $>19.8$ & $0.81 \mathrm{~m}$ Tenagra II \\
\hline Dec $11.5479 \ldots$. & $1.84 \mathrm{hr}$ & $I_{\mathrm{C}}$ & $19.99 \pm 0.24$ & $0.81 \mathrm{~m}$ Tenagra II \\
\hline Dec $11.5525 \ldots \ldots \ldots \ldots \ldots \ldots \ldots$ & $1.95 \mathrm{hr}$ & $R_{\mathrm{C}}$ & $20.74 \pm 0.42$ & $0.81 \mathrm{~m}$ Tenagra II \\
\hline Dec $11.5566 \ldots \ldots \ldots \ldots \ldots \ldots \ldots$ & $2.05 \mathrm{hr}$ & $R_{\mathrm{C}}$ & $20.70 \pm 0.16$ & 0.65 m Gunma \\
\hline Dec $11.9583 \ldots \ldots \ldots \ldots \ldots \ldots \ldots$ & $11.7 \mathrm{hr}$ & $R_{\mathrm{C}}$ & $>22.0$ & $1.34 \mathrm{~m}$ Tautenburg \\
\hline Dec $11.9744 \ldots \ldots \ldots \ldots \ldots \ldots$ & $12.1 \mathrm{hr}$ & $I_{\mathrm{C}}$ & $>20.7$ & $1.34 \mathrm{~m}$ Tautenburg \\
\hline Dec $12.3883 \ldots \ldots \ldots \ldots \ldots \ldots$ & $22.0 \mathrm{hr}$ & $i^{*}$ & $23.02 \pm 0.12$ & $3.5 \mathrm{~m} \mathrm{ARC}$ \\
\hline Dec $13.4680 \ldots \ldots \ldots \ldots \ldots \ldots$ & $47.9 \mathrm{hr}$ & $V$ & $23.0 \pm 0.5$ & $1.0 \mathrm{~m}$ USNO \\
\hline Dec $28.4283 \ldots \ldots \ldots \ldots \ldots \ldots$ & 17.0 days & $i^{*}$ & $24.41 \pm 0.22$ & $3.5 \mathrm{~m} \mathrm{ARC}$ \\
\hline Mar $23.1335 \ldots \ldots \ldots \ldots \ldots \ldots$ & 102 days & $i^{*}$ & $24.51 \pm 0.29$ & $3.5 \mathrm{~m} \mathrm{ARC}$ \\
\hline
\end{tabular}

${ }^{\text {a }}$ Upper limits are $3 \sigma$.

${ }^{\mathrm{b}}$ Flux weighted using an iterated power-law index of $\alpha=-1.37$ (§ 2.1).

$(z=1.004$ for GRB 021211 [Vreeswijk et al. 2002; Della Valle et al. 2002] vs. $z=1.600$ for GRB 990123 [Hjorth et al. 1999]; Fox et al. 2003; Li et al. 2003; Pandey et al. 2003; Crew et al. 2003). If it were not for the rapid response of the GRB community, GRB 021211 might have been called a "dark burst": it faded from $R \approx 14 \mathrm{mag}$ at $\approx 90 \mathrm{~s}$ after the burst (Wozniak et al. 2002) to $R>21$ mag about $3 \mathrm{hr}$ later. Many bursts that would have been called "dark" in the BeppoSAX era are being and will be called "dim" in the HETE-2, International Gamma-Ray Astrophysics Laboratory (INTEGRAL), and Swift era due to faster responses.

Some authors have modeled GRB 021211 with an emphasis on its environment. Kumar \& Panaitescu (2003) argue that the GRB and afterglow were produced by the same shock and within this framework constrain physical parameters for both constantdensity and wind-swept media. Panaitescu \& Kumar (2004) consider the early time afterglows of both GRB 021211 and GRB 990123 in the context of reverse-forward shock (for both constantdensity and wind-swept media) and wind-bubble scenarios, and find that the reverse-forward shock scenario is preferred. Chevalier et al. (2004) argue for a wind-swept medium with the cooling break redward of the $R$ band and within this framework find wind densities that are low compared to Galactic Wolf-Rayet stars (see also Panaitescu \& Kumar 2004). Finally, Dado et al. (2003) model GRB 021211 within the framework of their cannonball model.

In $\S 2$ we present FUN GRB Collaboration observations of GRB 021211, which include the earliest filtered detections and color information for an afterglow in the pre-Swift era. In $\S 3$ we fit standard afterglow and extinction curve models to these and other groups' data and show that within the first few hours after the burst one and possibly two significant chromatic variations are superimposed on this "standard model" behavior. In $\S 4$ we compare our results to previous modeling results and discuss possible reasons for these chromatic variations, including the possibility that they are due to a dust echo. We also constrain physical parameters that describe the afterglow and surrounding medium for a variety of scenarios and discuss why GRB 021211's afterglow is so faint. We draw conclusions in $\S 5$.

\section{OBSERVATIONS}

Long-duration, X-ray rich GRB 021211 was detected by HETE-2's FREGATE, WXM, and SXC instruments on 2002
December 11 at 11:18:34 UTC (Crew et al. 2003). The initial spacecraft localization was $14^{\prime}$ in radius and reported in nearreal time, only $22 \mathrm{~s}$ after the burst. Ground analysis of the WXM and SXC data, reported 131 minutes after the burst, improved the localization to $2^{\prime}$ in radius and was consistent with the initial localization.

Fox \& Price (2002) announced the discovery of an $R \sim 18$ mag and fading, stationary point source in the error circle 53 minutes after the burst. While the pair labored, the robotic telescopes of three groups had already responded to the alert. For only the second time in the afterglow era, robotic telescopes extended the light curve of an afterglow back to within tens of seconds of the burst (Wozniak et al. 2002; Li et al. 2002, 2003; Park et al. 2002; this paper).

The dim and quickly fading afterglow soon grew too faint for small telescopes, and a possible host galaxy was detected (Lamb et al. 2002a, 2002b; McLeod et al. 2002) but later confirmed under better seeing conditions to be cleanly separated from the afterglow by 1."5 (Caldwell et al. 2002). Very Large Telescope (VLT) spectroscopy of the true host galaxy resulted in a measured redshift of $z=1.004 \pm 0.002$ (Vreeswijk et al. 2002; Della Valle et al. 2002). Late-time observations indicate both a rebrightening at the time expected for a supernova at $z \sim 1$, and a spectrum that resembles that of Type Ic SN 1994I (Fruchter et al. 2002; Della Valle et al. 2003).

\subsection{FUN GRB Collaboration Observations}

We summarize FUN GRB Collaboration observations of GRB 021211 in Table 1 . We have calibrated all of our measurements using the field calibration of Henden (2002).

Super-LOTIS imaged the entire GRB 021211 field in $R$ band beginning 143 and $309 \mathrm{~s}$ after the burst (Park et al. 2002). SuperLOTIS is a fully automated f/3.5 $0.6 \mathrm{~m}$ diameter Perkin-Elmer telescope on a Boller \& Chivens mount at Kitt Peak National Observatory. The camera is a $2048 \times 2048$ Loral CCD, which yields a large, $51^{\prime} \times 51^{\prime}$ field of view. Observations began automatically after receiving the HETE-2 alert via a socket connection to the GCN. Both exposures were $60 \mathrm{~s}$ in duration. The mean times that we list in Table 1 are flux weighted using an iterated power-law index of $\alpha=-1.37$, since the exposure time is comparable to the age of the burst, at least for the first exposure. This 
results in small shifts of 2.4 and $1.2 \mathrm{~s}$ in the mean times of these observations.

The $0.81 \mathrm{~m}$ Tenagra II telescope from Tenagra Observatories, Ltd. imaged the GRB 021211 field beginning 37 minutes after the burst. We obtained four sets of four images, each set in a $2 \times 2$ arrangement to cover the initial $28^{\prime}$ diameter localization and each in a different filter $\left(I_{\mathrm{C}} R_{\mathrm{C}} V B\right)$. We then repointed to the candidate afterglow of Fox \& Price (2002) and cycled through $I_{\mathrm{C}} R_{\mathrm{C}}$ thrice more. Of these, we combined the first two $I_{\mathrm{C}}$ and $R_{\mathrm{C}}$ images to optimize the signal to noise ratio $(\mathrm{S} / \mathrm{N})$, but the final two images were not usable due to the onset of morning. This resulted in three detections $\left(I_{\mathrm{C}} R_{\mathrm{C}} V\right)$, a limit $(B)$, and two more detections $\left(I_{\mathrm{C}} R_{\mathrm{C}}\right)$. We reduced the images using IRAF's CCDRED package and performed point-spread function (PSF) photometry using IRAF's DAOPHOT package.

We imaged the central $11^{\prime} \times 11^{\prime}$ of the initial $28^{\prime}$ diameter localization in $R_{\mathrm{C}}$ band beginning 85 minutes after the burst from Gunma Astronomical Observatory, located in Agatsuma, Gunma, Japan (Kinugasa et al. 2002). We used the f/12 $0.65 \mathrm{~m}$ diameter Cassegrain telescope, which is equipped with an Apogee AP8 $1024 \times 1024$ back-illuminated SITe CCD. We obtained a total of 28 images, which we combined to optimize S/N. We reduced the images using IRAF's CCDRED package and performed PSF photometry on the combined image using IRAF's DAOPHOT package.

We reacquired the field with the $1.34 \mathrm{~m}$ diameter Tautenburg Schmidt telescope $11.7 \mathrm{hr}$ after the burst and imaged in $R$ and $I$ bands for the next $1.1 \mathrm{hr}$ using the $2048 \times 2048$ prime-focus CCD (Klose et al. 2002). However, we did not detect the afterglow.

We began observations with the $3.5 \mathrm{~m}$ diameter Astrophysics Research Consortium (ARC) telescope at Apache Point Observatory $22.0 \mathrm{hr}$ after the burst, and returned to the field on 2002 December 28 and 2003 March 23, 17 and 102 days after the burst (Lamb et al. 2002a, 2002b). All images were taken in $i^{*}$ band using SPIcam, a $2048 \times 2048$ back-illuminated SITe CCD. Three $2000 \mathrm{~s}$ images were taken on the first night, and seven $1200 \mathrm{~s}$ images were taken on each of the following nights. We reduced, combined, and calibrated these images using IRAF's CCDRED and DAOPHOT packages.

Finally, we reobserved the field on 2002 December 13 with the $1.0 \mathrm{~m}$ diameter telescope at the US Naval Observatory's Flagstaff Station for purposes of calibration (Henden 2002). $B V R_{\mathrm{C}} I_{\mathrm{C}}$ images were taken with a $2048 \times 2048$ back-illuminated SITe/ Tektronix CCD under 2".2 seeing conditions. Upon inspection of the images, the afterglow was still marginally visible in the 8 minute $V$-band image. The afterglow was measured using a twoFWHM diameter aperture with IRAF's DAOPHOT package.

\subsection{Implications of Late-Time ARC Observations}

Supernova signatures had been found for many GRBs prior to GRB 021211 (e.g., Galama et al. 1998, 2000; Bloom et al. 1999, 2003; Reichart 1999; Garnavich et al. 2003; Price et al. 2003; Stanek et al. 2003; see also Zeh et al. 2004 for a systematic analysis). For GRB 021211, Fruchter et al. (2002) and Della Valle et al. (2003) found evidence for excess red light $\approx 25$ days after the burst, and Della Valle et al. (2003) obtained a VLT spectrum at 27 days. This spectrum exhibits $\mathrm{Ca}$ II absorption with a relative velocity of $\approx 14,440 \mathrm{~km} \mathrm{~s}^{-1}$ for $z=1.004$ and is similar to other Type Ic spectra.

Our late-time ARC observations neither confirm nor contradict the existence of this underlying supernova. Subtraction of our second and third $i^{*}$ epochs using ISIS2 (Alard 2000) does not reveal any residual flux. However, this is likely due to the timing of our observations; the rebrightening reported by Della Valle

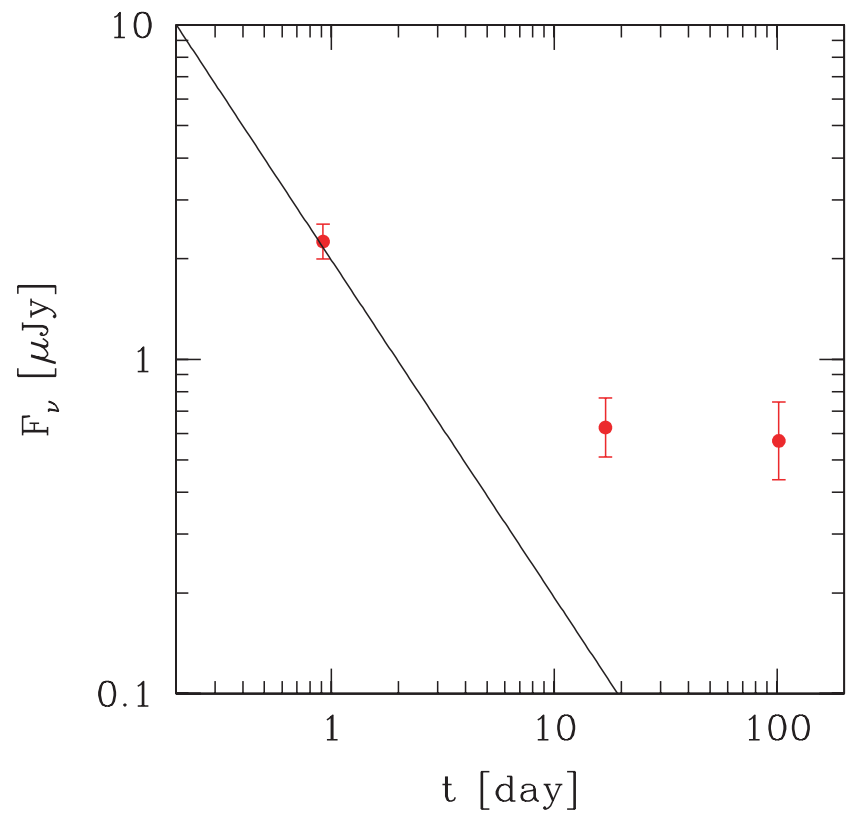

FIG. 1.-The $i^{*}$ light curve from $22 \mathrm{hr}$ to 102 days after the burst and best-fit WIND-BLUE model from $\S 3.1$. The host galaxy dominates at late times. We do not detect the supernova (Fruchter et al. 2002; Della Valle et al. 2003), likely due to the timing of our observations.

et al. (2003) occurs mostly between our observations at 17 and 102 days after the burst. In Figure 1 we plot our $i^{*}$ light curve and the fitted afterglow model of $\S 3.1$.

\subsection{Recalibration of KAIT and NEAT Photometry}

To better investigate possible chromatic variations that occurred during the unfiltered KAIT and Near-Earth Asteroid Tracking (NEAT) observations (see $\S 3.2$ ), we have recalibrated these measurements from the $R_{\mathrm{C}}$ band to broad bands given by the spectral responses of their respective CCDs (Pravdo et al. 1999; Li et al. 2003): W. Li (2003, private communication) and P. Price (2003, private communication) kindly provided us with their calibration stars. Using the $B V R_{\mathrm{C}} I_{\mathrm{C}}$ magnitudes of these stars from Henden (2002), we fitted extinguished blackbody functions to each of these stars and then integrated these fitted functions against the appropriate spectral response curve. This resulted in small, 0.05 and 0.03 mag offsets in the calibration of the KAIT and NEAT measurements, respectively.

\section{ANALYSIS}

We now fit standard afterglow and extinction curve models to these and other groups' data and show that within the first few hours after the burst, one and possibly two significant chromatic variations are superimposed on this "standard model" behavior. The data that we include in this analysis are plotted in Figure 2 and consist of FUN GRB Collaboration data ( $(2.1)$, data previously published in peer-reviewed journals (Pandey et al. 2003; Fox et al. 2003; Li et al. 2003; Holland et al. 2004), and data from the GCN archive (McLeod et al. 2002). These data span the first $\approx 2.5$ days after the burst, after which the host galaxy and supernova become contaminants. All magnitudes have been converted to spectral fluxes as prescribed by Bessell (1979) and Bessell \& Brett (1988).

\subsection{Model and Fits}

We now model these data and constrain model parameters. We model the afterglow with two components, corresponding 

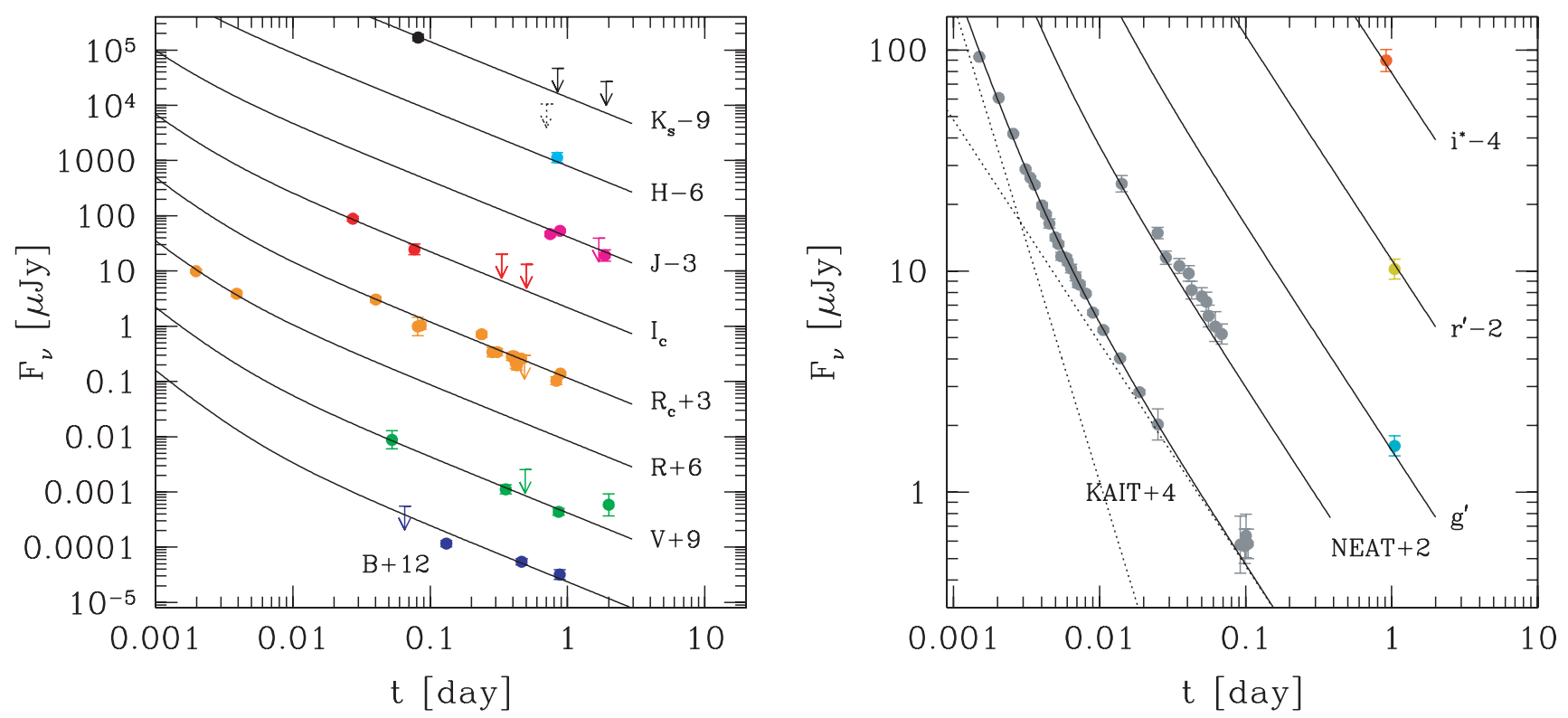

FIG. 2.-Left: $B V R R_{\mathrm{C}} I_{\mathrm{C}} J H K_{s}$ light curves from 2.8 minutes to 2.0 days after the burst and best-fit WIND-BLUE model from $\S 3.1$. Upper limits are $3 \sigma$. We do not include the dotted $K_{s}$ upper limit in our fits. Data are from McLeod et al. (2002), Pandey et al. (2003), Fox et al. (2003), Holland et al. (2004), and this paper. Right: $g^{\prime} r^{\prime} i^{*}$ and unfiltered KAIT and NEAT light curves from 9.2 minutes to 1.0 days after the burst and best-fit WIND-BLUE model from $\S 3.1$. The dotted curves are the reverse and forward shock components of the best-fit model for the spectral response of KAIT's CCD. Data are from Fox et al. (2003), Li et al. (2003), and this paper.

to reverse and forward shocks. Each component has a powerlaw light curve and a power-law spectrum, but the spectrum is extinguished by dust in the source frame and in our Galaxy and absorbed by hydrogen in the source frame and the $\operatorname{Ly} \alpha$ forest:

$$
\begin{aligned}
F_{\nu}(t)= & e^{-\tau_{\nu}^{\mathrm{MW}}} e^{-\tau_{\nu(1+z)}^{\mathrm{Ly} \alpha} e^{-\tau_{\nu(1+z)}^{\text {source }}}} F_{0}\left[\left(\frac{t}{t_{0}}\right)^{\alpha_{\mathrm{rs}}}\left(\frac{\nu}{\nu_{R}}\right)^{\beta_{\mathrm{rs}}}\right. \\
& \left.+\left(\frac{t}{t_{0}}\right)^{\alpha_{\mathrm{fs}}}\left(\frac{\nu}{\nu_{R}}\right)^{\beta_{\mathrm{fs}}}\right]
\end{aligned}
$$

where $\tau_{\nu}^{\mathrm{MW}}$ is the Galactic extinction curve model of Cardelli et al. (1989), $\tau_{\nu(1+z)}^{\mathrm{Ly} \alpha}$ is the Ly $\alpha$ forest absorption model of Reichart (2001a), $\tau_{\nu(1+z)}^{\text {soure }}$ is the source-frame extinction curve and Lyman limit absorption model of Reichart (2001a), $\alpha_{\mathrm{rs}}$ and $\alpha_{\mathrm{fs}}$ are the temporal indices of the two components, $\beta_{\mathrm{rs}}$ and $\beta_{\mathrm{fs}}$ are the spectral indices of the two components, $\nu_{R}$ is the effective frequency of the $R$ band, $t_{0}$ is the time when these two components are of equal brightness at this frequency, and $F_{0}$ is this brightness. Since the extinction and absorption models have features that are narrower than most photometric bands, we integrate equation (1) against the appropriate filter transmissivity curve (or CCD spectral response curve for the unfiltered measurements; $\S 2.3$ ) before fitting it to the data.

We fit this model to the data using Bayesian inference (e.g., Reichart 2001a; Lee et al. 2001; Galama et al. 2003); the posterior probability distribution is equal to the product of the prior probability distribution and the likelihood function. The likelihood function is given by

$$
\mathcal{L}=\prod_{i=1}^{N} \frac{1}{\sqrt{2 \pi\left(\sigma_{i}^{2}+\sigma^{2}\right)}} \exp \left\{-\frac{1}{2} \frac{\left[y\left(\nu_{i}, t_{i}\right)-y_{i}\right]^{2}}{\sigma_{i}^{2}+\sigma^{2}}\right\},
$$

where $N$ is the number of measurements, $y\left(\nu_{i}, t_{i}\right)$ is the above described integration of equation (1) against the spectral curve of the $i$ th measurement at the time of the $i$ th measurement; $y_{i}$ is the $i$ th measurement in units of log spectral flux; $\sigma_{i}$ is the uncertainty in the $i$ th measurement in the same units, and $\sigma$ is a parameter, sometimes called the slop parameter, that models the small systematic errors that are unavoidably introduced when data are collected from many sources and other small sources of error (Reichart 2001a). Ignoring this parameter can lead to erroneous fits and significantly underestimated uncertainties in the fitted parameter values when the scatter of the measurements about the fitted model exceeds that which can be accounted for by the measurement uncertainties alone.

Many of the parameters of the source-frame extinction curve model and all of the parameters of the Ly $\alpha$ forest absorption and Galactic extinction curve models can be constrained a priori. The source-frame extinction curve model of Reichart (2001a) is a function of eight parameters: the source-frame $V$-band extinction magnitude $A_{V}, R_{V}=A_{V} / E(B-V)$, the intercept $c_{1}$ and slope $c_{2}$ of the linear component of the source-frame UV extinction curve, the strength $c_{3}$, width $\gamma$, and center $x_{0}$ of the UV bump component of the extinction curve, and the strength $c_{4}$ of the FUV excess component of the extinction curve. The Ly $\alpha$ forest absorption model of Reichart (2001a) is a function of a single parameter, $D_{A}$, the flux deficit. Reichart (2001a) determines prior probability distributions for $R_{V}, c_{1}, \gamma, x_{0}$, and $D_{A}$, which means that the values of these parameters can be weighted by fairly narrow distributions, the description of which sometimes depends on other parameters $\left(c_{2}\right.$ and $\left.z\right)$, a priori. We adopt these priors here, which can be thought of as increasing the degrees of freedom by 5 . Also, the Galactic extinction curve model of Cardelli et al. (1989) is a function of $E(B-V)=0.028 \mathrm{mag}$ for this line of sight (Schlegel et al. 1998) and a single parameter, $R_{V}^{\mathrm{MW}}$. We adopt a prior for this parameter that is log normally distributed with mean $\log 3.1$ and width 0.1 , which closely approximates the distribution of values of this parameter along random lines of sight through the Galaxy (e.g., Reichart 2001a; Lee et al. 2001; Galama et al. 2003).

We fit our model to the data for each of the four standard cases of Sari et al. (1998) and Chevalier \& $\operatorname{Li}(2000)$, which relate $\alpha_{\mathrm{fs}}$ to 
$\beta_{\mathrm{fs}}$ assuming (1) propagation through either a constant-density (ISM) or wind-swept (WIND) medium and (2) a cooling break that is either redward (RED) or blueward (BLUE) of the observed optical and NIR frequencies: for the ISM-RED and WIND-RED cases, $\alpha_{\mathrm{fs}}=\left(3 \beta_{\mathrm{fs}}+1\right) / 2=-(3 p-2) / 4$; for the ISM-BLUE case, $\alpha_{\mathrm{fs}}=3 \beta_{\mathrm{fs}} / 2=-3(p-1) / 4$; and for the WIND-BLUE case, $\alpha_{\mathrm{fs}}=\left(3 \beta_{\mathrm{fs}}-1\right) / 2=-(3 p-1) / 4$, where $p$ is the powerlaw index of the electron-energy distribution. Since the temporal index is well constrained by the data, these additional constraints can be powerful tools for separating the intrinsic spectrum from extinction effects (see $\S 4.1$ ). For purposes of comparison, we also fit our model to the data, free of constraints on $\alpha_{\mathrm{fs}}$ and $\beta_{\mathrm{fs}}$.

The best fits are found by maximizing the posterior. Compared to the WIND-BLUE case, we can rule out the ISM-RED and WIND-RED cases at the $7.3 \sigma$ credible level, and the ISM-BLUE case is disfavored at the $3.1 \sigma$ credible level. Furthermore, the WIND-BLUE fit is consistent with the constraint-free fit, differing from it at only the $0.6 \sigma$ credible level. The primary difference between these cases is that the WIND-BLUE case requests a shallow intrinsic spectrum, $\beta_{\mathrm{fs}}=-0.34_{-0.01}^{+0.01}$, and a small amount of extinction, $A_{V}=0.18_{-0.12}^{+0.25} \mathrm{mag}$, where the other cases request steeper intrinsic spectra and would fit better if $A_{V}<0$ mag were possible (see $\S 4$ ). For the WIND-BLUE case, we find that $\log F_{0}(\mu \mathrm{Jy})=2.98_{-0.12}^{+0.12}, \log t_{0}($ days $)=-2.56_{-0.07}^{+0.07}, \alpha_{\mathrm{rs}}=$ $-2.16_{-0.10}^{+0.09}, \beta_{\mathrm{rs}}=1.1_{-0.8}^{+0.7}, \alpha_{\mathrm{fs}}=-1.01_{-0.01}^{+0.02}, \beta_{\mathrm{fs}}=-0.34_{-0.01}^{+0.01}$, $A_{V}=0.18_{-0.12}^{+0.25} \mathrm{mag}, c_{2}<4.3(1 \sigma)$, and $\sigma=0.038_{-0.008}^{+0.010} \mathrm{mag}^{19}{ }^{19}$ The parameters $c_{3}$ and $c_{4}$ could not be constrained by the data. We plot best-fit light curves for 13 spectral bands in Figure 2 and best-fit spectral flux distributions for six epochs in Figure 4.

\subsection{Chromatic Variations}

We plot the residuals of Figure 2 in Figure 3. One and possibly two significant chromatic variations can be seen from $\approx 40$ minutes after the burst until possibly $\approx 6.0 \mathrm{hr}$ after the burst. The first of these is an increase relative to the best-fit model of the unfiltered NEAT and KAIT data, which is also clearly visible in Figure 2 (right), concurrent with a decrease relative to the best-fit model of our $R_{\mathrm{C}}$ and possibly $I_{\mathrm{C}}$ data from Tenagra and Gunma. Since the NEAT and KAIT bandpasses are broad, encompassing the $I_{\mathrm{C}}$ and $R_{\mathrm{C}}$ bands on their red ends, this suggests that there was an excess of blue light at this time. To explore this further, we plot the spectral flux distribution of the afterglow in six time slices in Figure 4.

In Figure 4 (top left) we plot the best-fit spectral flux distribution at 67 minutes after the burst and have scaled all of the data between 39 and 94 minutes after the burst to this time using the best-fit light curve. These data consist of $I_{\mathrm{C}} R_{\mathrm{C}} V B$ data from Tenagra and unfiltered NEAT data. We plot the weighted average of the scaled NEAT data for clarity. The combined NEAT point is only 0.14 mag above the best-fit model, but significantly so, at the $5.2 \sigma$ confidence level.

In Figure 4 (top right) we plot the best-fit spectral flux distribution at $2.2 \mathrm{hr}$ after the burst. We have scaled all of the data between 1.8 and $2.5 \mathrm{hr}$ after the burst to this time using the bestfit light curve and plot weighted averages of the scaled data when there are multiple points per spectral band. These data consist of a $K_{s}$ point from Fox et al. (2003), $I_{\mathrm{C}} R_{\mathrm{C}}$ data from Tenagra, an $R_{\mathrm{C}}$ point from Gunma, and unfiltered KAIT data. The combined KAIT point is even farther above the best-fit model, $0.35 \mathrm{mag}$,

\footnotetext{
19 Due to the dimension of the parameter space, marginalized probability distributions for each parameter value would take impossibly long to compute. Consequently, these error bars are measured from projected probability distributions and are consequently conservative overestimates.
}

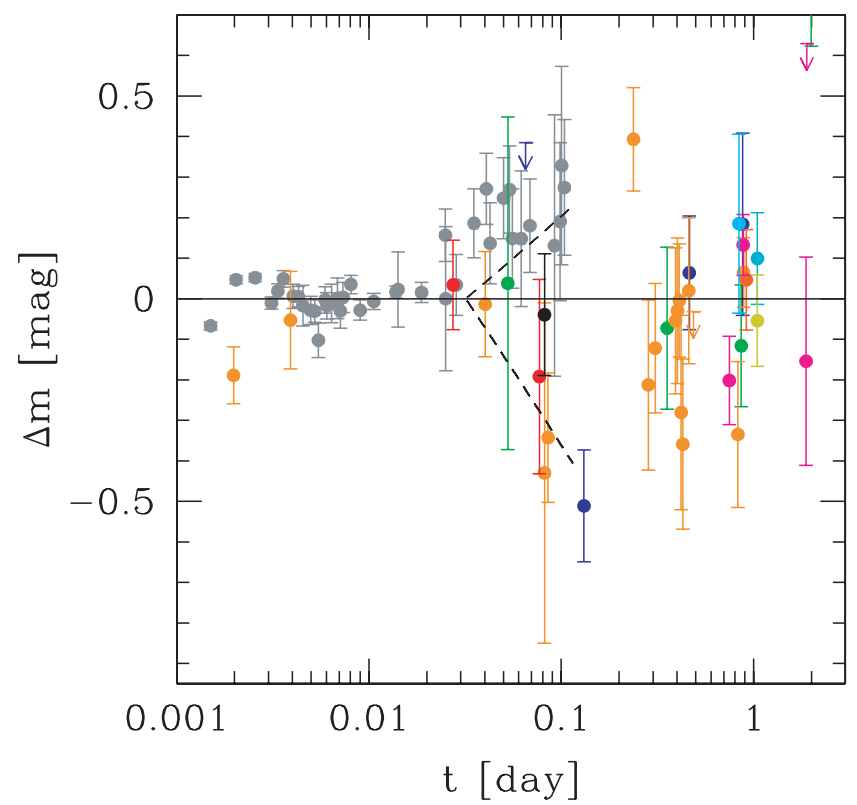

FIG. 3.-Residuals of Fig. 2. Colors are the same as in Fig. 2. Notice the increase relative to the best-fit model of the unfiltered NEAT and KAIT data, which is also clearly visible in Fig. 2 (right), concurrent with a decrease relative to the best-fit model of our $R_{\mathrm{C}}$ and possibly $I_{\mathrm{C}}$ data from Tenagra and Gunma. The dashed curves are our best-fit simple model for this from $\S 3.2$.

this time at the $3.3 \sigma$ confidence level. The $I_{\mathrm{C}}$ point is now below but still consistent with the best-fit model and the $R_{\mathrm{C}}$ point is below the best-fit model at the $2.3 \sigma$ confidence level. Consequently, the KAIT point differs from the $R_{\mathrm{C}}$ point at the $4.0 \sigma$ confidence level with respect to the best-fit model. Since the KAIT bandpass, like the NEAT bandpass, is broad, encompassing the $I_{\mathrm{C}}$ and $R_{\mathrm{C}}$ bands on its red end, this suggests that there was an excess of blue light at this time. If we model this excess as an additional power-law component, just in this time slice, we find it to be bluer than $\beta=1.0$ at the $2 \sigma$ credible level.

In Figure 4 (middle left) we plot the best-fit spectral flux distribution at $4.4 \mathrm{hr}$ after the burst. We have scaled two points from Fox et al. (2003), a $B$ point at $3.1 \mathrm{hr}$ after the burst and an $R_{\mathrm{C}}$ point at $5.7 \mathrm{hr}$ after the burst, to this time using the best-fit light curve. One possibility is that the excess light has changed from blue to red: the $R_{\mathrm{C}}$ point is above the best-fit model at the $3.1 \sigma$ confidence level, and the $B$ point is below the best-fit model at the $3.7 \sigma$ confidence level. Consequently, these points differ at the $4.8 \sigma$ confidence level with respect to the best-fit model. However, given the sparsity of the data in this time slice, a temporal variation cannot be ruled out either. The remaining panels, corresponding to $6.8-11,17-25$, and $46-48 \mathrm{hr}$ after the burst, show no evidence for significant chromatic variations at later times.

Although the third time slice is too sparsely sampled for a temporal variation to be ruled out, the first two time slices, which span the first proposed chromatic variation, are better sampled. Consider the following simple model. Let $t_{1}$ be the beginning of this variation. Prior to $t_{1}$, the afterglow is described by equation (1). Between $t_{1}$ and $2.5 \mathrm{hr}$ after the burst, the NEAT and KAIT data are instead described by temporal index $\alpha_{\mathrm{NK}}$ and the $R_{\mathrm{C}}$ and $I_{\mathrm{C}}$ data are instead described by temporal index $\alpha_{R_{\mathrm{C}} I_{\mathrm{C}}}$. If this were a temporal variation, $\alpha_{\mathrm{NK}}$ would equal $\alpha_{R_{\mathrm{C}} I_{\mathrm{C}}}$. Instead, we find that $t_{1}=46_{-21}^{+14}$ minutes and $\alpha_{\mathrm{NK}}-\alpha_{R_{\mathrm{C}} I_{\mathrm{C}}}=0.46_{-0.19}^{+0.23}$ with $\alpha_{\mathrm{NK}}-\alpha_{R_{\mathrm{C}} I_{\mathrm{C}}}>0$ at the $3.5 \sigma$ credible level. Here we have fixed all of the other parameters to their previous best-fit values so we can also plot this best fit in Figure 3. Allowing all of the 


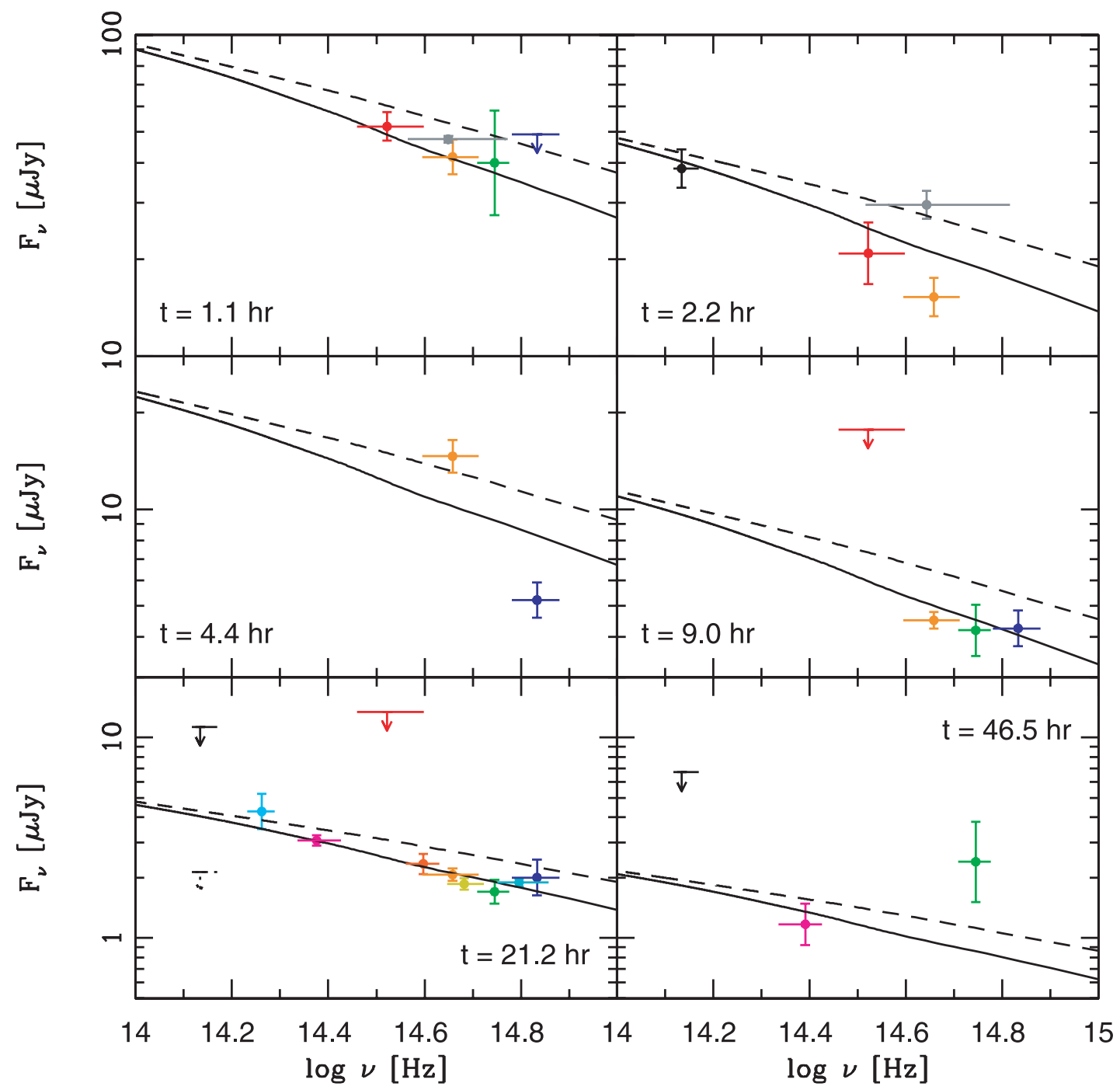

Fig. 4.- Spectral flux distribution at six epochs and best-fit WIND-BLUE model from $\S 3.1$ (solid curves). Dashed curves are the same fit, but with source-frame extinction set to zero. We scale data to these times using the best-fit light curve, and when there are multiple points per spectral band, we plot weighted averages of the scaled data for clarity (see $\S 3.2$ ). Colors are the same as in Fig. 2. Horizontal bars mark the $90 \%$ width of the filter. Upper limits are $3 \sigma$. We do not include the dotted $K_{s}$ upper limit in our fits.

parameters to vary, we find that $\alpha_{\mathrm{NK}}-\alpha_{R_{\mathrm{C}} I_{\mathrm{C}}}>0$ at the $3.3 \sigma$ credible level, which again suggests that this is a chromatic variation.

In the above fit, we also find that $\alpha_{R_{\mathrm{C}} I_{\mathrm{C}}}=-1.30_{-0.26}^{+0.20}$, which is somewhat steeper than the fitted value of $\alpha_{\mathrm{fs}}$. This suggests that the light curve might be steepening during the first and second time slices, and this is consistent with the $B$ point at the beginning of the third time slice also undercutting the model (e.g., Fig. 4, middle left). However, the data are consistent with the model in the fourth, fifth, and sixth time slices, which suggests a minor rebrightening during the third time slice. Such minor temporal variations are now commonplace, GRBs 021004 and 030329 are extreme examples, but further modeling of such variations is beyond the scope of this paper, and frankly beyond the quality of this data set. However, this does lend some credibility to the possibility that the second variation is temporal instead of chromatic. A final possibility is that the $R$ point at the end of the third time slice is a statistical variation: Given 80 points, the probability of encountering a $4.8 \sigma$ variation is 1 in 7900 (ruled out at the $3.8 \sigma$ confidence level).

Finally, we refit the four standard cases to the data, but this time we accommodate the first chromatic variation with the above simple model and eliminate the second variation, whether chromatic or temporal, by not fitting to the two points of the third time slice. Compared to the WIND-BLUE case, we now rule out the ISM-RED and WIND-RED cases at the $6.3 \sigma$ credible level and the ISM-BLUE case at the $3.2 \sigma$ credible level. For the WIND-BLUE case, we find that $\log F_{0}(\mu \mathrm{Jy})=2.27_{-0.25}^{+0.26}$, $\log t_{0}$ (days) $=-2.01_{-0.16}^{+0.17}, \alpha_{\mathrm{rs}}=-1.78_{-0.08}^{+0.07}, \beta_{\mathrm{rs}}=-0.95_{-0.55}^{+1.45}$, $\alpha_{\mathrm{fs}}=-0.89_{-0.04}^{+0.04}, \beta_{\mathrm{fs}}=-0.26_{-0.03}^{+0.03}, A_{V}=0.35_{-0.17}^{+0.22} \mathrm{mag}, c_{2}<$ $1.6(1 \sigma), t_{1}=7.5_{-3.5}^{+2.7}$ minutes, $\alpha_{R_{\mathrm{C}} I_{\mathrm{C}}}=-0.88_{-0.08}^{+0.08}, \alpha_{\mathrm{NK}}-$ $\alpha_{R_{\mathrm{C}} I_{\mathrm{C}}}=0.12_{-0.06}^{+0.06}$, and $\sigma=0.028_{-0.007}^{+0.007}$ mag. The primary difference between this fit and the WIND-BLUE fit of $\S 3.1$, in which these variations are not treated, is that we now find more source-frame extinction, $A_{V}=0.35_{-0.17}^{+0.22} \mathrm{mag}$ with $A_{V}>0 \mathrm{mag}$ at the $2.8 \sigma$ credible level. Also, $\alpha_{R_{C} I_{C}}$ is now consistent with $\alpha_{\mathrm{fs}}$, but $\alpha_{\mathrm{NK}}-\alpha_{R_{\mathrm{C}} I_{\mathrm{C}}}$ is still $>0$ at the $3.0 \sigma$ credible level.

\section{DISCUSSION}

\subsection{Model and Fits}

Our finding that the data are best described by the WINDBLUE case differs from the findings of others. Fox et al. (2003) discount this case in favor of the ISM-BLUE case, arguing that 
if the early-time emission is due to a reverse shock, in a windswept medium it is expected to fade quickly, and they measure a slower fading, $\alpha_{\mathrm{rs}}=-1.63 \pm 0.13$. However, Chevalier et al. (2004) point out that this measurement depends sensitively on how one subtracts out (or models) the forward-shock component, arguing that the value is closer to $\alpha_{\mathrm{rs}}=-2.2$. Using final instead of GCN data, we find that $\alpha_{\mathrm{rs}}=-2.16_{-0.10}^{+0.09}$ (variations untreated; $\S 3.1$ ) or $-1.78_{-0.08}^{+0.07}$ (variations treated; $\S 3.2$ ). However, in $\S 4.2$ we point out that emission from the reverse shock is not necessarily expected to fade quickly in a wind-swept me$\operatorname{dium}$ if $A_{*}$ and other physical parameters are lower than expected, which appears to be the case for this GRB.

Holland et al. (2004) also adopt the ISM-BLUE case. The primary difference between their fit and ours is that we permit source-frame extinction. When we fit the ISM-BLUE case, we find that $\beta_{\mathrm{fs}}=-0.67$ (variations untreated) or -0.60 (variations treated) with $A_{V}=0 \mathrm{mag}$, which is very similar to their fit in a time slice around 0.88 days after the burst: $\beta_{\mathrm{fs}}=-0.69 \pm 0.14$ with $A_{V}$ assumed to be zero. However, if source-frame extinction is permitted and one fits to all of the data, we find that the WIND-BLUE case with a small to moderate amount of sourceframe extinction, $A_{V}=0.18_{-0.12}^{+0.25} \mathrm{mag}$ (variations untreated) or $0.35_{-0.17}^{+0.22} \mathrm{mag}$ (variations treated), is preferred at the $3.1 \sigma$ (variations untreated) or $3.2 \sigma$ (variations treated) credible level. Figure 4 (bottom left) can be directly compared to Figure 3 of Holland et al. (2004).

Finally, Chevalier et al. (2004) adopt the WIND-RED case, guided by sparse color information that was available at the time, including the two points of Figure 4 (middle left), which we have already identified as discrepant, possibly due to excess red light at this time ( $\S 3.2)$. Permitting source-frame extinction and fitting to all of the data, we rule this case out at the $7.3 \sigma$ (variations untreated) or $6.3 \sigma$ (variations treated) credible level.

The WIND-BLUE case, however, requires a relatively hard electron energy distribution, $p=1.68_{-0.03}^{+0.01}$, so a break at higher energies is required. Bhattacharya (2001) determines the effect of $p<2$ on the standard equations by introducing a cutoff frequency $\gamma_{u}$ such that $\gamma_{m}<\gamma_{e}<\gamma_{u}$ and assuming that $\gamma_{u}$ evolves directly with the bulk Lorentz factor of the shock, they find results similar to the standard prescriptions. Galama et al. (2003) found a similar hard electron energy index for GRB 010222, although other ideas, such as a continuous injection of energy (Björnsson et al. 2002) or an early transition to nonrelativistic motion (in 't Zand et al. 2001; Masetti et al. 2001), have been proposed.

\subsection{Physical Parameters}

Following the analysis of Chevalier et al. (2004), but for the WIND-BLUE case, and using the analytic expressions of Granot $\&$ Sari (2002), we now constrain physical parameters that describe the afterglow and surrounding medium for a variety of scenarios. The first constraint comes from the expression of Granot \& Sari (2002) for the brightness of the afterglow in the frequency range of our observations, which for the WIND-BLUE case is $\max \left(\nu_{\mathrm{sa}}\right.$, $\left.\nu_{m}\right)<\nu<\nu_{c}$, where $\nu_{\mathrm{sa}}$ is the self-absorption frequency, $\nu_{m}$ is the typical synchrotron frequency, and $\nu_{c}$ is the electron cooling frequency. This corresponds to segment $G$ in their Figure 1. For $p=1.68$, a luminosity distance of $d_{L}=2.06 \times 10^{28} \mathrm{~cm}$ (assuming that $\Omega_{m}=0.3, \Omega_{\Lambda}=0.7$, and $\left.H_{0}=70 \mathrm{~km} \mathrm{~s}^{-1} \mathrm{Mpc}^{-1}\right)$, and an extinction-corrected $F_{R}=19 \mu \mathrm{Jy}$ at 0.1 days after the burst, we find

$$
\bar{\epsilon}_{e}^{0.68} \epsilon_{B}^{0.67} A_{*} E_{52}^{0.67}=1.02 \times 10^{-5},
$$

where $\bar{\epsilon}_{e}$ is the electron energy fraction when $p<2, \epsilon_{B}$ is the magnetic field energy fraction, $A_{*}$ measures the density of the wind-swept medium, and $E=E_{52} \times 10^{52}$ ergs is the total energy of the shock, if spherical. The second constraint comes from the expression of Granot \& Sari (2002) for $\nu_{c}(t)$ and the fact that the data are well described by the WIND-BLUE case even at early times (see $\S 4.3$ ). Taking $\nu_{c}>\nu_{R}$ prior to 3.9 minutes after the burst - the time when the forward shock first outshines the reverse shock in the $R$ band-yields

$$
\epsilon_{B}^{3 / 2} A_{*}^{2} E_{52}^{-1 / 2}=6.52 \times 10^{-6}\left(\frac{t_{c, R}}{3.9 \text { minutes }}\right)^{1 / 2},
$$

where $t_{c, R}$ is the time that $\nu_{c}$ passes above the $R$ band. The third constraint is similar to the second in that we take $\nu_{m}<\nu_{R}$ prior to 3.9 minutes after the burst, else the light curve would have faded much more slowly at this time, as $F_{\nu} \sim t^{-1 / 4}$ (Chevalier \& Li 2000; Chevalier et al. 2004),

$$
E_{52}^{1 / 2} \bar{\epsilon}_{e}^{2} \epsilon_{B}^{1 / 2}=1.18 \times 10^{-5}\left(\frac{t_{m, R}}{3.9 \text { minutes }}\right)^{3 / 2}
$$

where $t_{m, R}$ is the time when $\nu_{m}$ passes below the $R$ band. The final constraint comes from the expression of Granot \& Sari (2002) for the brightness of the afterglow at $8.5 \mathrm{GHz}$, given that $F_{8.5}<35 \mu \mathrm{Jy}$ at a mean time of 13 days after the burst (Fox et al. 2003). Here we consider four scenarios: (A) $\nu_{\mathrm{sa}}<8.5 \mathrm{GHz}<$ $\nu_{m}$, (B) $8.5 \mathrm{GHz}<\min \left(\nu_{\mathrm{sa}}, \nu_{m}\right)$, (C) $\max \left(\nu_{\mathrm{sa}}, \nu_{m}\right)<8.5 \mathrm{GHz}$, and (D) $\nu_{m}<8.5 \mathrm{GHz}<\nu_{\mathrm{sa}}$.

For scenario A, using the expression of Granot \& Sari (2002) that corresponds to their segment $\mathrm{D}$, we find:

$$
\bar{\epsilon}_{e}^{-2 / 3} \epsilon_{B}^{1 / 3} A_{*} E_{52}^{1 / 3}=3.76 \times 10^{-2}\left(\frac{F_{8.5}}{35 \mu \mathrm{Jy}}\right) .
$$

Combining equations (3), (4), (5), and (6) yields

$$
\begin{gathered}
\bar{\epsilon}_{e}=6.53 \times 10^{-4} E_{52}^{-1}\left(\frac{t_{c, R}}{3.9 \text { minutes }}\right)^{-0.25}\left(\frac{t_{m, R}}{3.9 \text { minutes }}\right)^{0.24} \\
\epsilon_{B}=765 E_{52}^{3}\left(\frac{t_{c, R}}{3.9 \text { minutes }}\right)\left(\frac{t_{m, R}}{3.9 \text { minutes }}\right)^{2.04} \\
A_{*}=1.75 \times 10^{-5} E_{52}^{-2}\left(\frac{t_{c, R}}{3.9 \text { minutes }}\right)^{-0.5}\left(\frac{t_{m, R}}{3.9 \text { minutes }}\right)^{-1.53} \\
F_{8.5}=20\left(\frac{t_{m, R}}{3.9 \text { minutes }}\right)^{-1.01} \mu \mathrm{Jy} .
\end{gathered}
$$

If we additionally require that $\nu_{\mathrm{sa}}<8.5 \mathrm{GHz}<\nu_{m}$ at 13 days after the burst, using the expressions of Granot \& Sari (2002) for $\nu_{\mathrm{sa}}$ and $\nu_{m}$, we find that $F_{8.5}<6 \mu \mathrm{Jy}$ and $E_{52}>3.50 \times$ $10^{-3}\left(F_{8.5} / 35 \mu \mathrm{Jy}\right)^{1.38}$. Since the former of these constraints contradicts equation (10) for any value of $t_{m, R}<3.9$ minutes, we rule out this scenario.

For scenario B, using the expression of Granot \& Sari (2002) that corresponds to their segment $\mathrm{B}$, we find

$$
\bar{\epsilon}_{e} A_{*}^{-1} E_{52}=1.70 \times 10^{-4}\left(\frac{F_{8.5}}{35 \mu \mathrm{Jy}}\right) .
$$


Combining equations (3), (4), (5), and (11) yields

$$
\begin{aligned}
\bar{\epsilon}_{e}= & 0.306\left(\frac{t_{c, R}}{3.9 \text { minutes }}\right)^{-0.125} \\
& \times\left(\frac{t_{m, R}}{3.9 \text { minutes }}\right)^{1.125}\left(\frac{F_{8.5}}{35 \mu \mathrm{Jy}}\right)^{-0.5}, \\
\epsilon_{B}= & 7.45 \times 10^{-6}\left(\frac{t_{c, R}}{3.9 \text { minutes }}\right)^{0.625} \\
& \times\left(\frac{t_{m, R}}{3.9 \text { minutes }}\right)^{-0.615}\left(\frac{F_{8.5}}{35 \mu \mathrm{Jy}}\right)^{1.5}, \\
A_{*}= & 3.85\left(\frac{t_{c, R}}{3.9 \text { minutes }}\right)^{-0.25} \\
& \times\left(\frac{t_{m, R}}{3.9 \text { minutes }}\right)^{0.24}\left(\frac{F_{8.5}}{35 \mu \mathrm{Jy}}\right)^{-1}, \\
E_{52}= & 2.14 \times 10^{-3}\left(\frac{t_{c, R}}{3.9 \text { minutes }}\right)^{-0.125} \\
& \times\left(\frac{t_{m, R}}{3.9 \text { minutes }}\right)^{-0.885}\left(\frac{F_{8.5}}{35 \mu \mathrm{Jy}}\right)^{0.5} .
\end{aligned}
$$

If we additionally require that $8.5 \mathrm{GHz}<\min \left(\nu_{\mathrm{sa}}, \nu_{m}\right)$ at 13 days after the burst, we find that $t_{m, R}>2.9$ minutes, which is technically consistent with $t_{m, R}<3.9$ minutes, and $F_{8.5}>27 \mu \mathrm{Jy}$, which is technically consistent with $F_{8.5}<35 \mu \mathrm{Jy}$. However, this constrains these parameters' values to narrow ranges and by equation (15) implies a value for $E_{52}$ that is much too low, given that the isotropic-equivalent energy in gamma rays alone was $(1.0 \pm$ $0.1) \times 10^{52}$ ergs (Holland et al. 2004) or $1.68_{-0.27}^{+0.32} \times 10^{52} \mathrm{ergs}$ (Lamb et al. 2004). Assuming that the efficiency at which energy is converted to gamma rays is $\sim 20 \%$ (e.g., Beloborodov 2000), then $E_{52} \sim$ many. Consequently, we rule out this scenario as well.

For scenario C, using the expression of Granot \& Sari (2002) that corresponds to their segment $\mathrm{G}$, we find

$$
\bar{\epsilon}_{e}^{0.68} \epsilon_{B}^{0.67} A_{*} E_{52}^{0.67}=6.26 \times 10^{-5}\left(\frac{F_{8.5}}{35 \mu \mathrm{Jy}}\right) .
$$

Combining equations (3), (4), (5) and (16) yields

$$
\begin{aligned}
& \bar{\epsilon}_{e}= 6.53 \times 10^{-4} E_{52}^{-1}\left(\frac{t_{c, R}}{3.9 \text { minutes }}\right)^{-0.25} \\
& \times\left(\frac{t_{m, R}}{3.9 \text { minutes }}\right)^{0.24}, \\
& \epsilon_{B}=765 E_{52}^{3}\left(\frac{t_{c, R}}{3.9 \text { minutes }}\right)\left(\frac{t_{m, R}}{3.9 \text { minutes }}\right)^{2.04}, \\
& A_{*}=1.75 \times 10^{-5} E_{52}^{-2}\left(\frac{t_{c, R}}{3.9 \text { minutes }}\right)^{-0.5} \\
& \times\left(\frac{t_{m, R}}{3.9 \text { minutes }}\right)^{-1.53}, \\
& F_{8.5}=6 \mu \mathrm{Jy} .
\end{aligned}
$$

If we additionally require that $\max \left(\nu_{\mathrm{sa}}, \nu_{m}\right)<8.5 \mathrm{GHz}$ at 13 days after the burst, we find that $t_{m, R}<13$ minutes and $E_{52}>1.27 \times$ $10^{-3}$, neither of which are problematic. Taking $E_{52}$ to be as low as 4 and $t_{c, R}$ and $t_{m, R}$ to be as low as the duration of the burst $\left(T_{90}=2.41 \pm 0.15 \mathrm{~s}\right.$ in the $30-85 \mathrm{keV}$ band, in which $\nu F_{\nu}$ peaks; Crew et al. 2003) yields $\epsilon_{B} \sim 0.04$. In this case, $\epsilon_{e} \sim$ 0.0002 and $A_{*} \sim 0.01$. However, for $\epsilon_{B}$ to be this low requires considerable fine tuning: if $E_{52}$ is as high as 11, $t_{c, R}$ is as high as $54 \mathrm{~s}, t_{m, R}$ is as high as $11 \mathrm{~s}$, or lesser combinations of these three, $\epsilon_{B} \approx 1$. Consequently, $\epsilon_{B}$ is likely considerably more than 0.04 , in which case $\epsilon_{e}$ can be no more than a factor of 3 greater and is likely less, and $A_{*}$ can only be less.

Finally, for scenario D, using the expression of Granot \& Sari (2002) that corresponds to their segment A, we find

$$
\epsilon_{B}^{-1 / 4} A_{*}^{-1} E_{52}^{3 / 4}=8.60 \times 10^{-2}\left(\frac{F_{8.5}}{35 \mu \mathrm{Jy}}\right) .
$$

Combining equations (3), (4), (5), and (21) yields

$$
\begin{aligned}
\bar{\epsilon}_{e}= & 0.232\left(\frac{t_{c, R}}{3.9 \text { minutes }}\right)^{-0.125} \\
& \times\left(\frac{t_{m, R}}{3.9 \text { minutes }}\right)^{0.75}\left(\frac{F_{8.5}}{35 \mu \mathrm{Jy}}\right)^{-0.5} \\
\epsilon_{B}= & 1.71 \times 10^{-5}\left(\frac{t_{c, R}}{3.9 \text { minutes }}\right)^{0.625} \\
& \times\left(\frac{t_{m, R}}{3.9 \text { minutes }}\right)^{0.51}\left(\frac{F_{8.5}}{35 \mu \mathrm{Jy}}\right)^{1.5} \\
A_{*}= & 2.21\left(\frac{t_{c, R}}{3.9 \text { minutes }}\right)^{-0.25} \\
& \times\left(\frac{t_{m, R}}{3.9 \text { minutes }}\right)^{-0.51}\left(\frac{F_{8.5}}{35 \mu \mathrm{Jy}}\right)^{-1}, \\
E_{52}= & 2.82 \times 10^{-3}\left(\frac{t_{c, R}}{3.9 \text { minutes }}\right)^{-0.125} \\
& \times\left(\frac{t_{m, R}}{3.9 \text { minutes }}\right)^{-0.51}\left(\frac{F_{8.5}}{35 \mu \mathrm{Jy}}\right)^{0.5},
\end{aligned}
$$

If we additionally require that $\nu_{m}<8.5 \mathrm{GHz}<\nu_{\mathrm{sa}}$ at 13 days after the burst, we find that $t_{m, R}>2.9$ minutes, which is technically consistent with $t_{m . R}<3.9$ minutes, and $F_{8.5}>7$ $\mu \mathrm{Jy}$, which is consistent with $F_{8.5}<35 \mu \mathrm{Jy}$. Once again, this constrains these parameters' values to relatively narrow ranges and by equation (25) implies a value for $E_{52}$ that is much too low. Therefore, we rule out this scenario as well.

Consequently, we find that $\nu_{m}<\nu_{R}<\nu_{c}$ after $<3.9$ minutes after the burst and $\max \left(\nu_{\mathrm{sa}}, \nu_{m}\right)<8.5 \mathrm{GHz}$ around 13 days after the burst. In this scenario, $\bar{\epsilon}_{e}$ and $A_{*}$ are considerably lower than canonical values. Since $F_{R} \propto \bar{\epsilon}_{e}^{0.68} \epsilon_{B}^{0.67} A_{*} E_{52}^{0.67}$ (eq. [3]), both of these contribute to the faintness of the afterglow $(\S 1)$.

If we allow ourselves to be guided by the standard-energy result (Frail et al. 2001; Bloom et al. 2003), $E_{52}$ is also lower than what one might expect. For wind-swept media, the total energy released in gamma rays is typically measured to be many $\times 10^{50} \mathrm{ergs}$ (Bloom et al. 2003). Given that the isotropic-equivalent energy in gamma rays for GRB 021211 was $(1.0 \pm 0.1) \times 10^{52} \mathrm{ergs}$ (Holland et al. 2004) or $1.68_{-0.27}^{+0.32} \times 10^{52} \mathrm{ergs}$ (Lamb et al. 2004), this implies a jet opening/viewing angle of $\sim 20^{\circ}$, which is about three times the canonical value. Hence, $E_{52}$ is probably about an order of magnitude less than what one might have expected. Consequently, we find that GRB 021211's afterglow is faint for a combination of reasons: (1) a low fraction of energy in relativistic electrons, (2) a low density for the wind-swept medium, implying either a low mass-loss rate and/or a high wind velocity for the 
progenitor, (3) a wide opening/viewing angle for the jet, and possibly (4) moderate source-frame extinction (§ 3.2).

Furthermore, with $\epsilon_{B} / \bar{\epsilon}_{e}>200$ and $\epsilon_{B}$ likely much greater than 0.04 the jet appears to be significantly far from equipartition and magnetically dominated. This is similar to SN 1993J, for which the magnetic field energy density is $\sim 10^{4}$ times the relativistic particle energy density (Chandra et al. 2004), but dissimilar to SN 1998bw (Kulkarni et al. 1998) and SN 2002ap (Björnsson \& Fransson 2004), which appear to be near equipartition.

These findings are supported by the existence of the bright reverse shock; Fox et al. (2003) dismiss the possibility of a wind-swept medium because for canonical values of $\bar{\epsilon}_{e}^{\mathrm{rs}}, \epsilon_{B}^{\mathrm{rs}}, A_{*}$, and $E_{52}, \nu_{c}^{\text {rs }}$ is expected to be significantly less than $\nu_{m}^{\text {rs }}$, in which case the reverse shock is expected to fade away quickly and not be bright (Chevalier \& Li 2000). However, this is not the case when $\bar{\epsilon}_{e}^{\mathrm{rs}}, \epsilon_{B}^{\mathrm{rs}}$, and/or $A_{*}$ are sufficiently low. Taking $\bar{\epsilon}_{e}^{\mathrm{rs}} \sim \bar{\epsilon}_{e}$ and $\epsilon_{B}^{\mathrm{rs}} \sim \epsilon_{B}$ and substituting equations (17), (18), and (19) into equations 45 and 47 of Chevalier \& Li (2000), we find

$\nu_{c}^{\mathrm{rs}} / \nu_{m}^{\mathrm{rs}}=5.56 \times 10^{4} E_{52}^{3}\left(\frac{t_{m, R}}{3.9 \text { minutes }}\right)^{0.03}\left(\frac{t}{1 \text { minute }}\right)^{2}$,

for $X=0.75, \Delta_{10}=3$, and $\gamma_{3}=0.3$. For $t=1$ minute after the burst, corresponding to the beginning of the first detection of the reverse shock (Wozniak et al. 2002), $E_{52}$ need only be greater than $\sim 0.03$ for $\nu_{c}^{\text {rs }} \sim \nu_{m}^{\text {rs }}$.

\subsection{Chromatic Variations}

Globally, the data are well described by the model of $\S 3.1$, but superimposed on this global behavior are small but significant variations from $\approx 40$ minutes after the burst until possibly $\approx 6.0 \mathrm{hr}$ after the burst ( $\S 3.2$ ). This model does not explain these variations. It merely attempts to accommodate them with a higher value of the slop parameter, $\sigma=0.038_{-0.008}^{+0.010}$ mag. The slop parameter is a global measure of the scatter of the data around the model, beyond what can be accounted for by the data's error bars $(\S 3.1)$. Furthermore, at least the first of these variations appears to be chromatic, with a relative increase of blue light with respect to red light around 2 hrafter the burst, and possibly, but less certainly, a reversal of this $2 \mathrm{hr}$ later ( $\S 3.2$ ). When we modify the model and fit to better treat these variations, $\sigma$ decreases to $0.028_{-0.007}^{+0.007} \mathrm{mag}(\S 3.2)$.

One potential explanation for these variations is that we have undersampled a light curve that is varying in time in such a way as to mimic a chromatic effect. Indeed, the high-S/N light curves of GRBs 021004 and 030329 revealed temporal variations and a variety of explanations have been proposed, including variations in the density of the external medium (e.g., Lazzati et al. 2002), refreshed shocks (e.g., Granot et al. 2003), and patchy shells (e.g., Kumar \& Piran 2000). However, none of these occur on a sufficiently short timescale to explain the variation around $2 \mathrm{hr}$ after the burst (Fig. 3). However, a temporal variation cannot be ruled out for the possible reversal of this effect $2 \mathrm{hr}$ later $(\S 3.2)$.

Another potential explanation is that a spectral break, presumably the cooling break, is passing through our data around $2-4 \mathrm{hr}$ after the burst. Although this is difficult to reconcile with the blue excess of Figure 4 (top right), since the spectrum is supposed to be half of a spectral index steeper blueward of the cooling break, it is not necessarily inconsistent with the possible red excess of Figure 4 (middle left). However, the spectra in Figure 4 (top left, bottom left) would then differ by half of a spectral index, which would be noticeable.

Another potential explanation is that we observed a dust echo, light scattered by dust into the line of sight and received with a time delay due to the greater path length. Waxman \& Draine (2000) and Esin \& Blandford (2000) originally proposed dust echoes as an alternative explanation for the supernova-like components to the afterglows of GRB 980326 (Bloom et al. 1999) and GRB 970228 (Reichart 1999; Galama et al. 2000). Reichart (2001b) modeled and computed dust echo light curves and spectral flux distributions and found that while dust echoes can mimic supernova light curves they cannot mimic supernova spectral flux distributions, at least not near the spectral peak. Moran \& Reichart (2005) take the model of Reichart (2001b), and instead of applying it to dust shells of inner radius $\sim 10^{18} \mathrm{~cm}$, which is what is required to mimic supernova light curves, they apply it to dust shells of inner radius $\sim 10^{14}-10^{15} \mathrm{~cm}$, which is typical of latetype WC Wolf-Rayet stars, the likely progenitors of GRBs.

Moran \& Reichart (2005) find that for (1) wind-swept media, (2) bright optical flashes, reverse shocks that outshine the forward shock at early times, and (3) wide jet opening angles, dust echoes may be observable on a timescale of minutes to hours after the burst. Furthermore, the characteristic signature will be an excess of blue light (since blue light scatters preferentially) that quickly transitions to an excess of red light (due to increasing path lengths through dust with increasing time delay). Since all of these conditions appear to be met for GRB 021211 $(\S \S 3.1,4.1,4.2)$, and excess blue light is observed on this timescale, as well as a possible transition to excess red light hours later, we now test this hypothesis in two ways.

1. Equation (6) of Moran \& Reichart (2005) gives the turnon time of an idealized, on-axis dust echo as a function of the inner radius $R$ of the circumprogenitor dust shell and the initial opening angle $\theta_{\text {jet }}$ of the jet. Dust echoes should not be visible at very early times because X-rays from the burst and UV light from the optical flash should sublimate the dust within $\theta_{\text {jet }}$ of the jet axis this close to the burst (e.g., Waxman \& Draine 2000; Fruchter et al. 2001; Reichart 2001c). Taking the turn-on time to be $\sim 0.4$ of the peak time (Reichart 2001b; Moran \& Reichart 2005) and taking the peak time for GRB 021211 to be $\sim 2.2 \mathrm{hr}$, we solve for $R$,

$$
R \sim 3 \times 10^{15}\left(\frac{\theta_{\text {jet }}}{10^{\circ}}\right)^{-2} \mathrm{~cm}
$$

For a wide jet opening angle, this yields $R \sim 10^{14}-10^{15} \mathrm{~cm}$, which is the expectation if late-type WC stars are indeed the progenitors of GRBs. However, given that the isotropic-equivalent energy in gamma rays alone was $(1.0 \pm 0.1) \times 10^{52}$ ergs (Holland et al. 2004) or $1.68_{-0.27}^{+0.32} \times 10^{52} \mathrm{ergs}$ (Lamb et al. 2004) and that $A_{*} \lesssim 0.01$ ( $\left.\S 4.2\right)$, the deceleration radius $r_{d}$ was likely greater than $10^{16} \mathrm{~cm}$ (e.g., eq. [7] of Moran \& Reichart 2005) and consequently a value for $R$ likely cannot be deduced.

2. Equation (5) of Moran \& Reichart (2005), but with $r_{d}$ substituted for $R$, gives the peak brightness of the optical flash off of the jet axis at angles around $\theta_{\text {jet }}$ as a function of the peak brightness of the dust echo, the deceleration radius, the optical depth $\tau_{\nu(1+z)}$ through the rest of the dust shell at frequency $\nu(1+z)$ also at angles around $\theta_{\text {jet }}$, and the duration of the optical flash. Taking the peak brightness of the dust echo to be $\sim 19$ mag and the duration of the optical flash to be $\sim 30$ s (e.g., Sari \& Piran 1999), we find

$m_{\mathrm{OF}}\left(\theta \sim \theta_{\text {jet }}\right) \sim 9+2.5 \log \tau_{\nu(1+z)}-2.5 \log \left(\frac{r_{d}}{10^{16} \mathrm{~cm}}\right)$ mag. 
Using our best fit ( $\S 3.1$ ), extrapolation of the reverse-shock light curve back to $\approx 30 \mathrm{~s}$ after the onset of the burst yields $R \sim$ 12 mag. However, given the known distance to GRB 021211, $m_{\mathrm{OF}}\left(\theta \sim \theta_{\text {jet }}\right)$ would have to be significantly fainter lest the dust be sublimated in these directions as well, this close to the burst. For longer optical flash durations, $\tau_{\nu(1+z)}>1$, and/or a greater forward scattering probability than what Esin \& Blandford (2000), Reichart (2001b), and Moran \& Reichart (2005) assume, $m_{\mathrm{OF}}\left(\theta \sim \theta_{\text {jet }}\right)$ would be fainter, but not sufficiently. Alternatively, prior fragmentation of the dust to polycyclic aromatic hydrocarbon (PAH) levels by gamma rays from the burst might harden it against sublimation, since atomic bonds would then be more difficult to break.

Finally, we point out that neither of these estimates hold in the case of a jet with a narrow opening angle but a large viewing angle.

\section{CONCLUSIONS}

GRB 021211 is one of only a handful of GRBs for which processes other than the forward shock have been identified at optical wavelengths, which has made it one of the most studied GRBs. In this paper we present additional, multiband observations of this event, ranging from minutes to months after the burst, which in combination with all previously published observations have allowed us to deeply probe the physics of this GRB and properties of its circumprogenitor environment.

Coupling the standard afterglow model with a general-purpose extinction curve model, we find that the afterglow is best described by propagation through a wind-swept medium, which implies a massive-star progenitor (e.g., Price et al. 2002). The jet itself appears to be significantly far from equipartition and magnetically dominated. Indeed, the low fraction of energy in relativistic electrons appears to be the primary reason that this afterglow is so faint. This, combined with a low-density medium, a wide jet opening/viewing angle, and possibly a moderate extinction might be important clues as to why many afterglows are dark/dim in the Swift era, even at early times after the burst. These findings are supported by the existence of the bright reverse shock, in a wind-swept medium this should only be possible if $A_{*}$ is low and/or the jet is significantly far from equipartition, meaning that either $\bar{\epsilon}_{e}^{\mathrm{rs}}$ or $\epsilon_{B}^{\mathrm{rs}}$ is low as well.

Finally, we observed one and possibly two significant chromatic variations hours after the burst. We discuss possible reasons for these variations, including the possibility that they are due to a dust echo. The three primary requirements for an observable dust echo are a wind-swept medium, a bright optical flash, and a wide jet opening angle, and the characteristic signature should be an excess of blue light that quickly reddens, all of which appear to be satisfied for GRB 021211. However, in the case of GRB 021211 this would imply an off-axis brightness and hence luminosity for the optical flash that would probably sublimate too much dust in these directions. Rapid, multiband, and preferably simultaneous multiband observations of future GRBs might shed more light on this interesting possibility.

D. E. R. very gratefully acknowledges support from NSF's MRI, CAREER, PREST, and REU programs, NASA's APRA, Swift GI and IDEAS programs, UNC's Junior Faculty Development Award, and especially Leonard Goodman and Henry Cox. H. S. P. acknowledges support from NASA's Supporting Research and Technology program and DOE/UC LLNL contract W-7405-Eng-48. K. K. acknowledges support from Ministry of Education, Culture, Sports, Science, and Technology of Japan grant-in-aid 16740121. D. Q. L. acknowledges support from NASA subcontract NAGW-4690 (HETE-2) and NASA's LTSA program.
Akerlof, C., et al. 1999, Nature, 398, 400

Alard, C. 2000, A\&AS, 144, 363

Beloborodov, A. M. 2000, ApJ, 539, L25

Bessell, M. S. 1979, PASP, 91, 589

Bessell, M. S., \& Brett, J. M. 1988, PASP, 100, 1134

Bhattacharya, D. 2001, Bull. Astron. Soc. India, 29, 107

Björnsson, C., \& Fransson, C. 2004, ApJ, 605, 823

Björnsson, G., Hjorth, J., Pedersen, K., \& Fynbo, J. U. 2002, ApJ, 579, L59

Bloom, J. S., Frail, D. A., \& Kulkarni, S. R. 2003, ApJ, 594, 674

Bloom, J. S., et al. 1999, Nature, 401, 453

Caldwell, N., McLeod, B., Garnavich, P., \& Stanek, K. Z. 2002, GCN Circ., 1759,1

Cardelli, J. A., Clayton, G. C., \& Mathis, J. S. 1989, ApJ, 345, 245

Chandra, P., Ray, A., \& Bhatnagar, S. 2004, ApJ, 612, 974

Chevalier, R. A., \& Li, Z. 2000, ApJ, 536, 195

Chevalier, R. A., Li, Z., \& Fransson, C. 2004, ApJ, 606, 369

Crew, G., et al. 2002, GCN Circ., 1734, 1 2003, ApJ, 599, 387

Dado, S., Dar, A., \& De Rújula, A. 2003, ApJ, 593, 961

Della Valle, M., et al. 2002, GCN Circ., 1809, 1 2003, A\&A, 406, L33

Esin, A. A., \& Blandford, R. 2000, ApJ, 534, L151

Fox, D. W., \& Price, P. A. 2002, GCN Circ., 1731, 1

Fox, D. W., et al. 2003, ApJ, 586, L5

Frail, D. A., et al. 2001, ApJ, 562, L55

Fruchter, A., Krolik, J. H., \& Rhoads, J. E. 2001, ApJ, 563, 597

Fruchter, A., Levan, A., Vreeswijk, P., Holland, S. T., \& Kouveliotou, C. 2002,

GCN Circ., 1781, 1

Galama, T. J., et al. 1998, Nature, 395, 670

2000, ApJ, 536, 185

2003, ApJ, 587, 135

Garnavich, P. M., et al. 2003, ApJ, 582, 924

Granot, J., Nakar, E., \& Piran, T. 2003, Nature, 426, 138

\section{REFERENCES}

Granot, J., \& Sari, R. 2002, ApJ, 568, 820

Henden, A. 2002, GCN Circ., 1753, 1

Hjorth, J., et al. 1999, GCN Circ., 219, 1

Holland, S. T., et al. 2004, AJ, 128, 1955

in 't Zand, J. J. M., et al. 2001, ApJ, 559, 710

Kinugasa, K., Kato, T., Yamaoka, H., \& Torii, K. 2002, GCN Circ., 1749, 1

Klose, S., Eisloeffel, J., Froebrich, D., Zeh, A., Richter, S., Hoegner, C., \& Voss, H. 2002, GCN Circ., 1739, 1

Kulkarni, S. R., et al. 1998, Nature, 395, 663

Kumar, P., \& Panaitescu, A. 2003, MNRAS, 346, 905

Kumar, P., \& Piran, T. 2000, ApJ, 535, 152

Lamb, D. Q., Barentine, J. C., Nysewander, M. C., Reichart, D. E., Schwartz, M., Laws, C., York, D. G., \& McMillan, R. J. 2002a, GCN Circ., 1744, 1

Lamb, D. Q., Nysewander, M. C., Reichart, D. E., Barentine, J. C., Laws, C., York, D. G., \& McMillan, R. J. 2002b, GCN Circ., 1765, 1

Lamb, D. Q., et al. 2004, NewA Rev., 48, 423

Lazzati, D., Rossi, E., Covino, S., Ghisellini, G., \& Malesani, D. 2002, A\&A, 396, L5

Lee, B. C., et al. 2001, ApJ, 561, 183

Li, W., Filippenko, A. V., Chornock, R., \& Jha, S. 2002, GCN Circ., 1737, 1 2003, ApJ, 586, L9

Masetti, N., et al. 2001, A\&A, 374, 382

McLeod, B., Caldwell, N., Grav, T., Luhman, K., Garnavich, P., \& Stanek, K. Z. 2002, GCN Circ., 1750, 1

Moran, J., \& Reichart, D. 2005, ApJ, 632, 438

Panaitescu, A., \& Kumar, P. 2004, MNRAS, 353, 511

Pandey, S. B., Anupama, G. C., Sagar, R., Bhattacharya, D., Castro-Tirado, A. J., Sahu, D. K., Parihar, P., \& Prabhu, T. P. 2003, A\&A, 408, L21

Park, H. S., Williams, G., \& Barthelmy, S. 2002, GCN Circ., 1736, 1

Pravdo, S. H., et al. 1999, AJ, 117, 1616

Price, P. A., et al. 2002, ApJ, 572, L51 2003, ApJ, 589, 838

Reichart, D. E. 1999, ApJ, 521, L111 
Reichart, D. E. 2001a, ApJ, 553, 235 2001b, ApJ, 554, 643

Reichart, D. E. 2001c, preprint (astro-ph/0107546)

Sari, R., \& Piran, T. 1999, ApJ, 520, 641

Sari, R., Piran, T., \& Narayan, R. 1998, ApJ, 497, L17

Schlegel, D. J., Finkbeiner, D. P., \& Davis, M. 1998, ApJ, 500, 525

Smith, I. A., et al. 2005, A\&A, 439, 987
Stanek, K. Z., et al. 2003, ApJ, 591, L17

Vreeswijk, P., Burud, I., Fruchter, A., \& Levan, A. 2002, GCN Circ., 1767, 1

Waxman, E., \& Draine, B. T. 2000, ApJ, 537, 796

Wei, D. M. 2003, A\&A, 402, L9

Wozniak, P., et al. 2002, GCN Circ., 1757, 1

Zeh, A., Klose, S., \& Hartmann, D. H. 2004, ApJ, 609, 952 